\title{
Disorder effects in electronic structure of substituted transition metal compounds
}

\author{
D.D. Sarmat, A. Chainanit and S.R. Krishnakumar \\ Solid State and Structural Chemistry Unit, Indian Institute of Science, Bangalore 560 012, INDIA \\ E. Vescovo, C. Carbone and W. Eberhardt \\ Institut für Festkörperforschung, Forschungszentrum Jülich, D-52425 Jülich, GERMANY \\ O. Rader, Ch. Jung, Ch. Hellwig and W. Gudat \\ BESSY, Lentzeallee 100, D-14195 Berlin, GERMANY \\ H. Srikanth and A.K. Raychaudhuri \\ Department of Physics, Indian Institute of Science, Bangalore 560 012, INDIA
}

(May 22, 2018)

\begin{abstract}
Investigating $\mathrm{LaNi}_{1-x} M_{x} \mathrm{O}_{3}(M=\mathrm{Mn}$ and $\mathrm{Fe})$, we identify a characteristic evolution of the spectral function with increasing disorder in presence of strong interaction effects across the metal-insulator transition. We discuss these results vis-a-vis existing theories of electronic structure in simultaneous presence of disorder and interaction.
\end{abstract}

The last few years have seen a spectacular resurgence of interest in investigating the phenomena of metal-insulator transitions (MIT) in substituted transition metal oxides (TMO). MIT which are driven by electron interaction effects as in the Mott-Hubbard picture 11.2) or by disorder effects causing an Anderson transition [3], have led to distinctly different paradigms. However, experimental realisations of MIT in substituted TMO's show signatures of both these effects. A few theoretical attempts [4 8] have been made to study the electronic structure in simultaneous presence of interaction and disorder. However, these do not provide a unifying description of the ground state electronic structure and the MIT. Briefly summarizing, Altshuler-Aronov (AA) theory for disordered metals 相 predicts a characterisitc $\left|E-E_{F}\right|^{1 / 2}$ cusp at $E_{F}$ progressively depleting the density of states (DOS) at the Fermi energy, $N\left(E_{F}\right)$, on approaching the MIT boundary from the metallic side. On the other hand, a disorder driven insulator would open up a soft Coulomb gap [0] characterised by a $\left(E-E_{F}\right)^{2}$ dependence of DOS near $E_{F}$ in presence of long range interactions. The AA theory, restricting itself to a narrow energy scale within the metallic state, or the Coulomb gap insulating phase, does not provide a description for either the evolution of the electronic structure across the transition, or the electronic structure over a larger energy scale $(\sim U$ or $W)$. Treating diagonal disorders in a Hubbard-like model within the dynamical mean field theory [9, it has been predicted [7,10] that the electronic structure of a substituted TMO resembles an additive average of the electronic structures of the two stoichiometric end-members on a wide energy scale. While this is the only prediction available for the evolution of electronic structures over a wide energy scale for disordered substituted TMO, it suggests the formation of a narrow resonance peak at $E_{F}$ within the metallic phase in contrast to the AA theory. Additionally, while these conflicting scenarios relate only to ground state electronic structures, experimentally various interesting cross-overs of transport properties are often observed as a function of temperature, suggesting changes in the electronic structure over a low energy scale with changing temperature 111.

In most cases of substituted TMO, the MIT is achieved by altering the composition via substitution chemistry, thereby tuning the charge carrier density by doping. While this way of doping charge carriers invariably introduces disorder in the system, this aspect is often ignored. This has led to the wide spread use of a homogeneous model like the Hubbard model which includes only interaction effects, to describe the evolution of electronic structures in these systems. However, in many cases there are obvious experimental signatures not understood within Hubbard-like models; for example, at very low temperatures, $\mathrm{La}_{2-x} \mathrm{Sr}_{x} \mathrm{CuO}_{4}$ with $x=0.02$ shows Coulomb gap behavior which changes to variable range hopping $(\mathrm{VRH})$ for $\mathrm{x}=0.05$ [12]. The need to dope macroscopic charge carrier concentrations (often upto 0.2 or even more per transition metal site) to drive the insulator-to-metal transition is also beyond the scope of any homogeneous model. However, disorder induced changes in the electronic structure are often difficult to probe in presence of chemical substitutions, since such substitutions result in large changes in the electronic structure due to the doping of new (electron or hole) states; these tend to dominate over every other subtle change in the electronic structure. Additionally, doping induced structural transitions can make it impossible to extricate the effects of disorder vis-a-vis correlations.

In order to study the evolution of electronic structure 
of a correlated system exhibiting a MIT due to static disorder but in absence of (i) charge carrier alteration in the form of doped states as well as (ii) a structural instability, we have carried out a detailed spectroscopic investigation of the isostructural series, $\mathrm{LaNi}_{1-x} M_{x} \mathrm{O}_{3}(M=\mathrm{Mn}, \mathrm{Fe}$ or Co). While $\mathrm{LaNiO}_{3}$ is a correlated metallic oxide [13], $\mathrm{La} \mathrm{O}_{3}$ with $M=\mathrm{Mn}, \mathrm{Fe}$ and $\mathrm{Co}$ are insulating and the solid solutions $\mathrm{LaNi}_{1-x} M_{x} \mathrm{O}_{3}$ exhibit [14] MIT with increasing $x$. In early work, this transition was discussed essentially in terms of percolation models, since the end members of the solid solution have differing ground state electronic properties. However percolation theory cannot obviously account for very different values of the critical concentration, $x_{c}$ with changing $M: x_{c}(\mathrm{Mn}) \approx 0.1$, $x_{c}(\mathrm{Fe}) \approx 0.2$ and $x_{c}(\mathrm{Co}) \approx 0.65$, for the same structure, with lattice parameters remaining more or less unaltered. Moreover, this is a case of homovalent substitution $\left(\mathrm{Ni}^{3+}\right.$ by $M^{3+}$ ) as against heterovalent substitution (e.g. $\mathrm{La}^{3+}$ by $\mathrm{Sr}^{2+}$, which dopes hole states). This makes the system ideally suited for investigating possible manifestations of disorder effects within the strongly interacting transition metal oxide system. We show that indeed there is a pronounced cusp in the DOS at low energy scales (within $200 \mathrm{meV}$ of $E_{F}$ ) in contrast to the resonance expected within the Hubbard-like model. Interestingly however, changes in the electronic structure over a wider energy scale is in agreement with this model, suggesting that the existing theories are only partially successful in describing the evolution of the electronic structure with changing disorder in such strongly correlated systems. Additionally, spectral functions exhibit changes that correlate with the cross-over of the transport behavior as a function of temperature.

The sample preparation and characterization have been described earlier [15]. Photoemission experiments were carried out at SX-700-II and 3M-NIM-I beamlines of BESSY using a high-resolution VG ESCALAB system with a resolution of about $100 \mathrm{meV}$. Bremsstrahlung isochromat spectra (BIS) were recorded in a VSW spectrometer with a resolution of about $0.7 \mathrm{eV}$. The samples were maintained at $100 \mathrm{~K}$ to avoid surface degradation and intermittently scraped in-situ to obtain a clean surface. Point-contact conductance spectra were obtained at $4.2 \mathrm{~K}$, using electrochemically etched gold tips.

We show BIS of $\mathrm{LaNi}_{1-x} \mathrm{Mn}_{x} \mathrm{O}_{3}$ in Fig. 1 which is dominated by transition metal $3 d$ states over the first $3 \mathrm{eV}$ with minimal overlap from other states. $\mathrm{LaNiO}_{3}$ spectrum clearly shows a large intensity, while $\mathrm{LaMnO}_{3}$ exhibits negligible intensity at $E_{F}$, consistent with the metallic and insulating properties respectively. It turns out that the spectra of the intermediate compositions can be well described by a weighted average of the spectra of the end-members $\left(\mathrm{LaNiO}_{3}\right.$ and $\left.\mathrm{LaMnO}_{3}\right)$, as shown by solid lines superimposed on the experimental data. This remarkable agreement over a wide energy scale is consistent with the dynamical mean-field result of a Hubbard- like model with diagonal disorder [7, 10. While similar conclusions can also be drawn on the basis of oxygen $K$-edge x-ray absorption spectra of all the three series, $\mathrm{LaNi}_{1-x} M_{x} \mathrm{O}_{3}$ (with $M=\mathrm{Mn}, \mathrm{Fe}$ or Co) 15], these observations cannot explain the occurrence of the MIT in the ground state across a finite value of $x$.

For the near-ground state low-energy scale electronic structure of these systems, we have obtained pointcontact conductance spectra of the series $\mathrm{LaNi}_{1-x} \mathrm{Mn}_{x} \mathrm{O}_{3}$ at $4.2 \mathrm{~K}$ (Fig. 2). Since the point contact tunneling conductance, to a first order, is given by the DOS at low temperatures and small bias voltages [16], these spectra provide a representation of the changes in the DOS with high resolution. The conductance of the metallic systems with $x \leq 0.1$ as a function of the applied voltage, $V$, clearly shows the typical cusp at $E_{F}$ for a disordered system. A linear plot of the conductance vs. $V^{1 / 2}$ (inset I of Fig. 2) establishes the characterisitic square-root dependence. Moreover, it is known that a cusped DOS at $E_{F}$ leads to a $T^{1 / 2}$ dependence of the conductivity at low temperatures $\sqrt{6}$. Such a $T^{1 / 2}$ dependence has indeed been observed [17] in these samples. This is in sharp contrast to the predictions based on the dynamical meanfield theory of the Hubbard-like model which predicts the formation of an increasingly narrow Kondo-like peak at $E_{F}$. These results on the spectral evolution with increasing disorder is directly relevant not only for the resistivity, but also for other physical properties such as specific heat, which depend on the DOS at $E_{F}$. Thus, while the dynamical mean-field theory appears to describe the large-energy scale behavior reasonably well, it fails to depict the actual changes in the low energy scale near $E_{F}$. For the semiconducting composition $(x=0.2)$, the conductance is linear in $V$ and shows the signature of a hard gap $(\approx 20 \mathrm{meV})$ at $E_{F}$. Qualitatively similar tunneling spectra have also been obtained [18] for $\mathrm{LaNi}_{1-x} \mathrm{Co}_{x} \mathrm{O}_{3}$. However, the transport measurements at higher temperatures for the insulating composition [17] do not reveal an activated behavior characteristic of a hard gap. Instead, variable range hopping is observed even at $10 \mathrm{~K}$ suggesting the existence of finite localised DOS at $E_{F}$. Interestingly, tunneling conductance measured at $10 \mathrm{~K}$ for $x=0.2$ sample exhibits a wiping out of the hard gap with a significant increase in the zero bias conductance as shown in the inset II.

In order to probe the nature of single particle spectral weights in the vicinity of $E_{F}$ at finite temperatures, we have studied the photoemission spectra of $\mathrm{LaNi}_{1-x} \mathrm{Mn}_{x} \mathrm{O}_{3}$ and $\mathrm{LaNi}_{1-x} \mathrm{Fe}_{x} \mathrm{O}_{3}$ series at $100 \mathrm{~K}$, with $h \nu=55 \mathrm{eV}$ shown in Fig. 3 over a narrow energy scale. The spectra of $\mathrm{LaMnO}_{3}$ [19] and $\mathrm{LaFeO}_{3}$ [20], being wide band gap $(\approx 1.3$ and $2.0 \mathrm{eV}$ respectively) materials, have no appreciable weight over the narrow energy range near $E_{F}$ and the spectral function at the intermediate compositions is dominated by the $\mathrm{LaNiO}_{3}$ component of the solid solution. The results show that there is a system- 
atic depletion of spectral weight within $\approx 200 \mathrm{meV}$ of $E_{F}$ with increasing $\mathrm{Mn}$ and Fe concentrations as is clearly evident in the insets. The spectral function modifications in the two series are very similar, indicating that the nature of changes in the spectral functions arises only from the disorder caused by the substitution, and follows the same qualitative behavior. In order to quantify the changes in the spectral function near $E_{F}$, we have first analysed the $\mathrm{LaNiO}_{3}$ spectrum in terms of a polynomial DOS varying smoothly across $E_{F}$, broadened by the resolution function and temperature (Fermi-Dirac statistics). The resulting fit is shown by solid lines through the data points in Fig. 3. Subsequently, we simulate the spectra of the metallic compositions with $x>0.0$ by a DOS that is identical to that obtained from $\mathrm{LaNiO}_{3}$ farther away from $E_{F}$ as suggested by the inset, but with a $\left|E-E_{F}\right|^{1 / 2}$ cusp near $E_{F}$ prompted by the depletion of spectral weights near $E_{F}$ in the photoemission spectra and the results in Fig. 2. We find that the simulated spectra (solid lines) describe the experimental results well. However, our attempt to describe the spectra of the insulating compositions in terms of a linear DOS near $E_{F}$ with a hard gap as suggested by the conductance spectra (Fig. 2) was not successful, as shown by dashed lines in Fig. 3. Clearly there is a lot more intensity at $E_{F}$ in the experimental curve to be compatible with a gap even after taking into account the resolution and thermal broadenings, suggesting a finite density of states at $E_{F}$ at elevated temperatures for the compositions with an insulating ground state (for $\mathrm{Mn}, x=0.2$, and for $\mathrm{Fe}$, $x=0.3$ and 0.4 ). Thus, we attempt to describe the spectral features for these compositions with a DOS identical to $\mathrm{LaNiO}_{3}$ in the wider energy scale with a $\left|E-E_{F}\right|^{m}$ dependence close to $E_{F}$ in order to represent the pronounced depletion of states there. We find that $m=1 / 2$ provides a good description also for insulating compositions as shown by the solid lines in the figures. We show the extracted DOS for all the cases without the resolution and thermal broadening in the insets II in Figs. 3a and $b$. These plots clearly show the formation of the cusp for the substituted compositions with a progressive depletion of states near $E_{F}$. At finite temperatures, the insulating compositions appear to be characterised by a deeper cusp existing over a wider energy scale. In every case, the extracted DOS farther away from $E_{F}$ is very similar to that of $\mathrm{LaNiO}_{3}$.

To summarize the present results vis-a-vis existing theories, we find that the ground state electronic structure within the metallic phase in the substituted compounds over a narrow energy scale is in agreement with AA theory [4] with a characteristic cusp at $E_{F}$. This is in contrast with the formation of a sharp resonance peak at $E_{F}$ expected on the basis of Hubbard-like models [7, 10]; we further point out that the expected resonance peak is absent even in the parent stoichiometric $\mathrm{LaNiO}_{3}$. On the other hand, the formation of a hard gap in the ground state on the insulating side of the MIT is not consistent with the soft Coulomb gap predicted for disordered insulating systems in presence of long range interactions and may indicate the dominance of on-site interactions as in the Hubbard model. It is interesting to note that the spectral evolution over a wide energy scale (ignoring the subtle changes close to $E_{F}$ ) is consistent with the prediction of the Hubbard-like model with diagonal disorder. In any case, it is clear that none of the existing theories is sufficient to describe entirely the evolution of the ground state electronic structure across the MIT in substituted TMO. The present results provide evidence of a strong influence of disorder on the electronic structure of substituted TMO. It also explains the systematic change in the value $\left(x_{c}\right)$ of critical substitution required for driving the MIT in this family of compounds, $\mathrm{LaNi}_{1-x} M_{x} \mathrm{O}_{3}$ (with $M=\mathrm{Mn}, \mathrm{Fe}$ or Co). As the substitutional element $M$ is farther away from $\mathrm{Ni}$ in the Periodic Table, the strength of the disorder as measured by the difference of the bare energies at the $\mathrm{Ni}$ and the $M$ sites, increases, requiring less number of disordered sites to drive the transition and leading to the decreasing trend of $x_{c}$ as observed. Additionally, the present results provide evidence of a wiping out of the ground state hard gap of the insulating compositions at elevated temperatures. It is a challenging theoretical task to provide a microscopic basis for such interesting dynamics of the gap with temperature. Even more fundamental is the question of the ground state metal-insulator transition in substitutional transition metal compounds with clear evidence for a simultaneous presence of strong interaction and disorder effects, since it is clear that no exisitng theory is able to explain all the experimentally observed changes in the electronic structure across the transition. Our results emphasize the need to include the on-site Coulomb interaction as in the Hubbard model within a theory of disorder like Altshuler-Aronov theory which captures the low-energy features of disorder effects. While such a theory has not been attempted so far, we believe that the present results will motivate efforts in this direction.

Acknowledgements : DDS thanks Forschungszentrum, Jülich for hospitality during a part of this work; AC and SRK thanks the CSIR, India for financial support. This research is funded by the Department of Science and Technology, Government of India.

* Also at Jawaharlal Nehru Centre for Advanced Scientific Research, Bangalore. Electronic address: sarma@sscu.iisc.ernet.in

$\dagger$ Permanent address: Institute for Plasma Research, Gandhinagar 382 428, India. 
[1] N.F. Mott, Proc. Phys. Soc. London, Sect. A 62, 416 (1949); N. F. Mott, Metal-Insulator Transitions (Taylor and Francis, London, 1990).

[2] J. Hubbard, Proc. Roy. Soc. London A 277, 237 (1964); J. Hubbard, ibid. 281, 401 (1964).

[3] P. W. Anderson, Phys. Rev. 109, 1492 (1958).

[4] B.L. Altshuler and A.G. Aronov, Solid State Commun. 30, 115 (1979).

[5] M. Pollak, Discuss. Faraday Soc. 50, 7 (1970); V. Ambegaokar,B. I. Halperin, and J. S. Langer, Phys. Rev. B 4, 2612 (1971); A. F. Efros and B. I. Shklovskii, J. Phys. C: Solid State Phys. 8, L49 (1975); J. G. Massey and Mark Lee, Phys. Rev. Lett. 75, 4266 (1995).

[6] P. A. Lee and T. V. Ramakrishnan, Rev. Mod. Phys. 57, 287 (1985).

[7] D. D. Sarma, S. R. Barman, H. Kajueter, and G. Kotliar, Europhys. Lett. 36, 307 (1996).

[8] V. Dobrosavljević and G. Kotliar, Phys. Rev. Lett. 78, 3943 (1997).

[9] A. Georges, G. Kotliar, W. Krauth, and M. Rozenberg, Rev. Mod. Phys. 68, 13 (1996).

[10] G. Kotliar, unpublished results.

[11] A. Husmann et al., Science 274, 1874 (1996); A. Matsuura et al., Phys. Rev. B 53, R7584 (1996); I. Terry et al., Phys. Rev. Lett. 69, 1800 (1992).

[12] T.F. Rosenbaum and. S.A. Carter, J. Solid State Chem. 88, 94 (1990).

[13] K.P. Rajeev, G. V. Shivashankar and A. K. Raychaudhuri, Solid State Comm. 79, 591 (1991); K. Sreedhar et al., Phys. Rev. B 46, 6382 (1992).

[14] P. Ganguly, N. Y. Vasanthacharya, C. N. R. Rao, and P. P. Edwards, J. Solid State Chem. 54, 400 (1984).

[15] D.D. Sarma et al., Phys. Rev. B 49, 14238 (1994).

[16] J. Tersoff and D.R. Hamann, Phys. Rev. Lett. 50, 1998 (1983); J.A. Stroscio et al., Phys. Rev. Lett. 75, 2960 (1995).

[17] A. Chainani, D. D. Sarma, I. Das, and E. V. Sampathkumaran, J. Phys. Condens. Matter 8, L631 (1996).

[18] K.P. Rajeev, Ph.D. thesis, Indian Institute of Science, Bangalore 1994.

[19] A. Chainani, M. Mathew, and D. D. Sarma, Phys. Rev. B 47, 15397 (1993); T. Saitoh et al., Phys. Rev. B 51, 13942 (1995).

[20] A. Chainani, M. Mathew, and D. D. Sarma, Phys. Rev. B 48, 14818 (1993).

Figure captions:

Fig. 1 BIS of $\mathrm{LaNi}_{1-x} \mathrm{Mn}_{x} \mathrm{O}_{3}$ (open circles). The synthesized spectra (solid lines) for intermediate compostions are generated by weighted average of the spetra of the end-members, $\mathrm{LaNiO}_{3}$ and $\mathrm{LaMnO}_{3}$; the relative weight of the $\mathrm{LaMnO}_{3}$ component in the synthesized spectra is shown as a function of $x$ by the open sircles in the inset, exhibiting a linear dependence as expected.

Fig. 2 Point-contact tunneling conductance of $\mathrm{LaNi}_{1-x} \mathrm{Mn}_{x} \mathrm{O}_{3}$. The inset $\mathrm{I}$ shows the conductance as a function of $\sqrt{V}$. The solid lines are guides to the eye. The inset II shows the comparison of tunneling conductance for $x=0.2$ sample at 4.2 and $10 \mathrm{~K}$, exhibiting a vanishing of the hard gap and an increase in the zero bias conductance.

Fig. 3 Photoemission spectra of $\mathrm{LaNi}_{1-x} M_{x} \mathrm{O}_{3}$ ((a) $M$ $=\mathrm{Mn}$ and (b) $M=\mathrm{Fe}$ ) with $h \nu=55 \mathrm{eV}$. Spectra are superposed in the inset I to illustrate the systematic depletion of states close to $E_{F}$ with increasing $x$. The full lines show a fit to simulate the depletion in the DOS with the corresponding DOS shown in inset II. The dashed lines are the best fits to the spectra assuming the DOS to be linear near $E_{F}$ with a hard gap of $20 \mathrm{meV}$. 


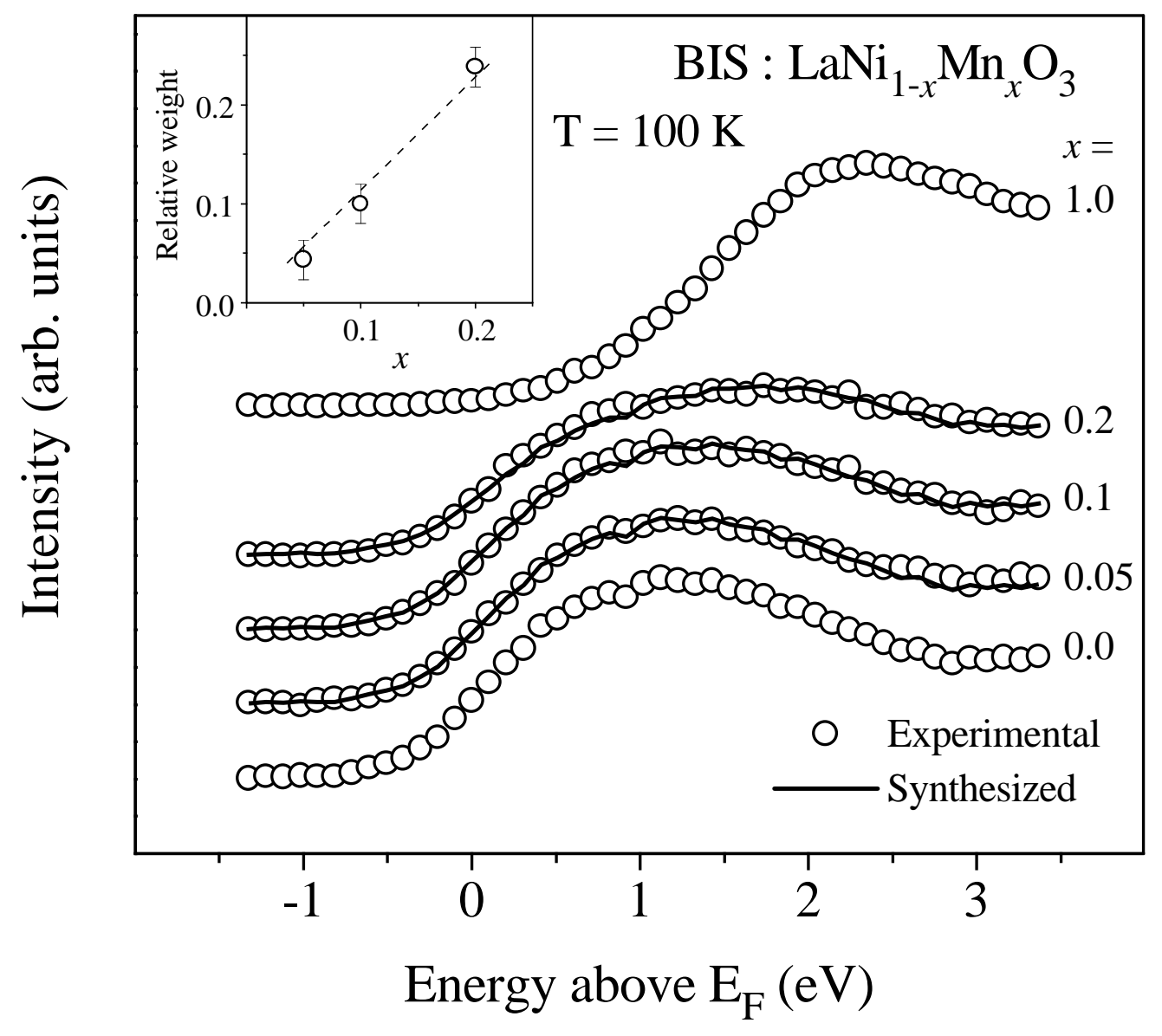

Sarma et al., Fig. 1 


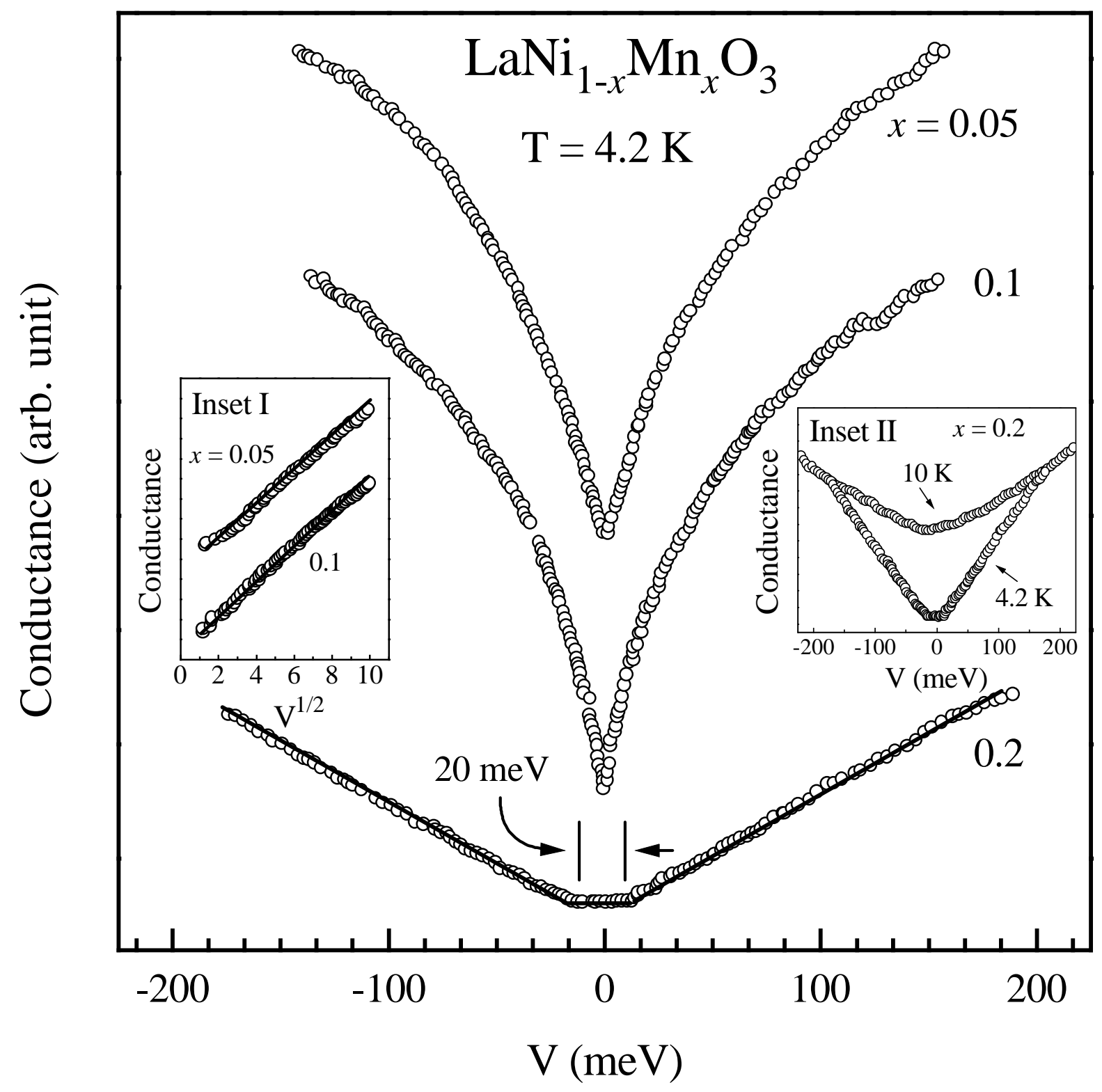

Sarma et al., Revised Fig. 2 


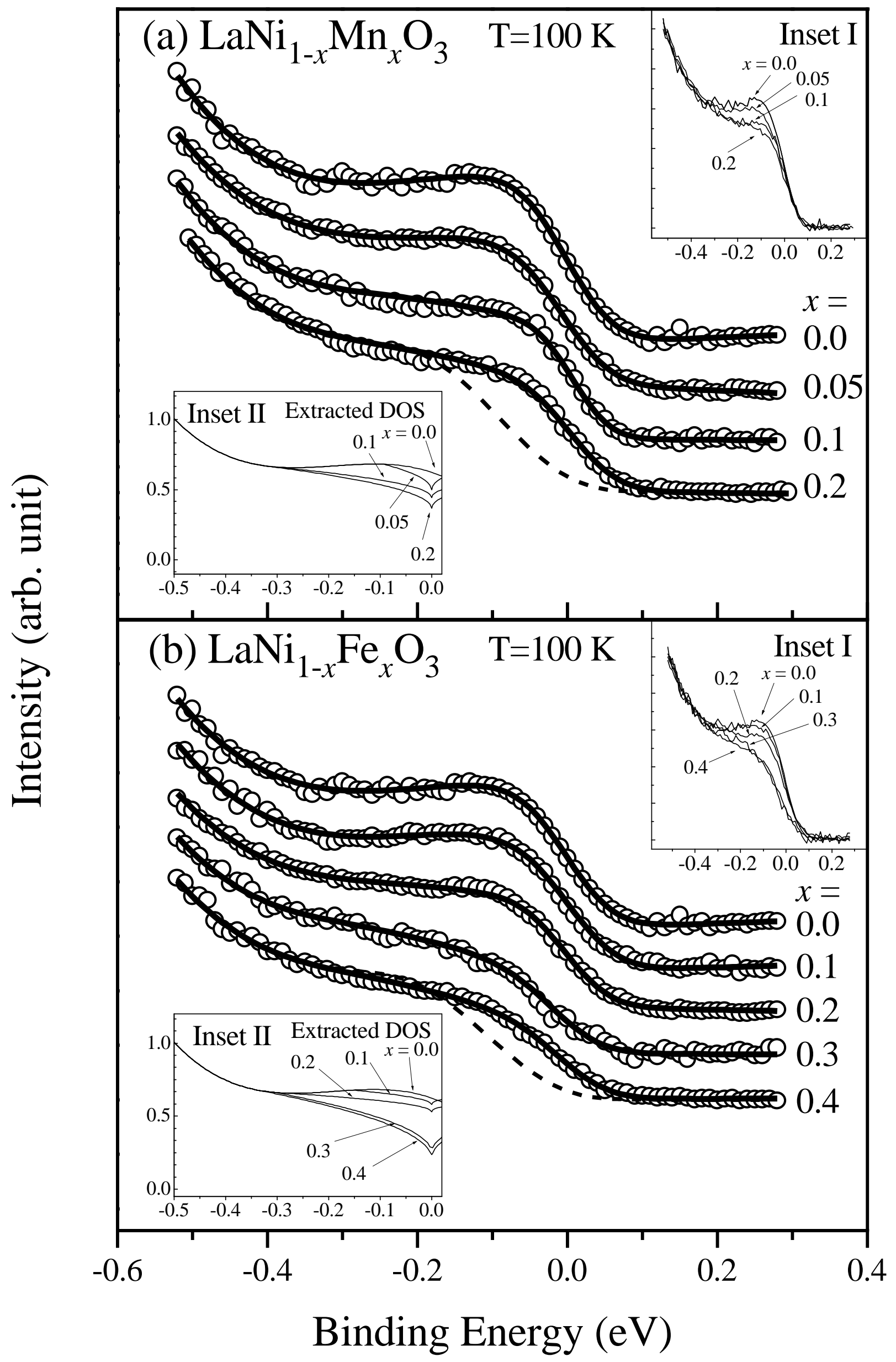

Sarma et al., Fig. 3 